\title{
Do lower extremity kinematics and training variables affect the development of overuse injuries in runners? - a prospective study
}

\author{
Tobias Hein *, Pia Janssen, Ursula Wagner-Fritz, Stefan Grau \\ From 3rd Congress of the International Foot and Ankle Biomechanics Community \\ Sydney, Australia. 11-13 April 2012
}

\section{Background}

The incidence of running injuries appears to be multifactorial, e.g. with regard to training errors or kinematics of the lower extremity [1,2]. To date, studies examining differences between healthy and injured runners are mainly retrospective, and therefore not able to determine whether these differences are the cause or effect of injury. The goal of this prospective study is to evaluate whether the development of overuse injuries in initially healthy subjects is caused by alterations in lower extremity kinematics and/or training habits.

\section{Materials and methods}

Since April 2009, 218 healthy runners $(m=139, w=79)$ have been included in the study. Besides a standardized clinical examination, 3D-kinematics of both lower extremities were recorded according to ISB-recommendations [3]. Hip, knee and ankle joint motions were quantified by calculating Cardan angles [4]. Mean angular displacements and discrete parameters were calculated from 10 valid trials of barefoot running. All subjects were asked to complete and return weekly training logs with information about their weekly mileage, running time, and type of training sessions, as well as information about the occurrence of pain during training. Study duration lasted a maximum of 12 months or until diagnosis of an overuse injury.

\section{Results}

Currently, 35 from 104 subjects who have completed the study have developed overuse injuries. Symptomatic runners ran more training kilometers per week than healthy runners, and showed a shift from slow and medium to fast endurance training sessions. Further, runners who generated overuse injuries exhibited differences in rear foot, ankle and knee kinematics compared to runners who remained uninjured during their participation.

\section{Conclusions}

The initial results indicate that lower leg kinematics and training parameters are risk factors and consequently possible causes for developing overuse injuries. Further subjects will be included in the evaluation to enable a division into larger injury-specific groups.

Published: 10 April 2012

\section{References}

1. van Gent RN, Siem D, van Middelkoop M, et al: Incidence and determinants of lower extremity running injuries in long distance runners: a systematic review. Br J Sports Med 2007, 41:469-480.

2. Willems $T M$, De CD, Delbaere $K$, Vanderstraeten $G$, et al: A prospective study of gait related risk factors for exercise-related lower leg pain. Gait Posture 2006, 23:91-98.

3. Wu G, Siegler $S$, Allard $P$, et al: ISB recommendation on definitions of joint coordinate system of various joints for the reporting of human joint motion-part I: ankle, hip, and spine. International Society of Biomechanics. J Biomech 2002, 35:543-548.

4. Grood ES, Suntay WJ: A joint coordinate system for the clinical description of three-dimensional motions: application to the knee. $J$ Biomech Eng 1983, 105:136-144.

doi:10.1186/1757-1146-5-S1-O46

Cite this article as: Hein et al:: Do lower extremity kinematics and training variables affect the development of overuse injuries in runners? - a prospective study. Journal of Foot and Ankle Research 20125 (Suppl 1):046.

\footnotetext{
* Correspondence: tobias.hein@med.uni-tuebingen.de

Department of Sports Medicine, Medical Clinic, University of Tübingen,

Tübingen, Germany
}

(c) 2012 Hein et al; licensee BioMed Central Ltd. This is an Open Access article distributed under the terms of the Creative Commons 